\title{
Is rat an appropriate animal model to study the involvement of D-serine catabolism in schizophrenia? insights from characterization of D-amino acid oxidase
}

\author{
Luca F. Frattini ${ }^{1,2}$, Luciano Piubelli ${ }^{1,2}$, Silvia Sacchi ${ }^{1,2}$, Gianluca Molla ${ }^{1,2}$ and Loredano Pollegioni ${ }^{1,2}$ \\ 1 Dipartimento di Biotecnologie e Scienze della Vita, Università degli studi dell'Insubria, Varese, Italy \\ 2 Centro Interuniversitario di Ricerca in Biotecnologie Proteiche 'The Protein Factory', Università degli studi dell'Insubria, Politecnico di \\ Milano, and ICRM-CNR, Milan, Italy
}

\author{
Keywords \\ drug design; D-serine; flavoproteins; ligand \\ binding; schizophrenia

\section{Correspondence \\ L. Pollegioni, Dipartimento di Biotecnologie e Scienze della Vita, Università degli studi dell'Insubria, via J.H. Dunant 3, 21100 \\ Varese, Italy \\ Fax: +39 332421500 \\ Tel: +39332421506 \\ E-mail: Ioredano.pollegioni@uninsubria.it}

(Received 7 June 2011, revised 12 September 2011, accepted 14 September 2011)

doi:10.1111/j.1742-4658.2011.08354.x
D-Amino acid oxidase (DAAO; EC 1.4.3.3) has been proposed to play a main role in the degradation of D-serine, an allosteric activator of the $N$-methyl-D-aspartate-type glutamate receptor in the human brain, and to be associated with the onset of schizophrenia. To prevent excessive D-serine degradation, novel drugs for schizophrenia treatment based on DAAO inhibition were designed and tested on rats. However, the properties of rat DAAO are unknown and various in vivo trials have demonstrated the effects of DAAO inhibitors on D-serine concentration in rats. In the present study, rat DAAO was efficiently expressed in Escherichia coli. The recombinant enzyme was purified as an active, $40 \mathrm{kDa}$ monomeric flavoenzyme showing the basic properties of the dehydrogenase-oxidase class of flavoproteins. Rat DAAO differs significantly from the human counterpart because: (a) it possesses a different substrate specificity; (b) it shows a lower kinetic efficiency, mainly as a result of a low substrate affinity; (c) it differs in affinity for the binding of classical inhibitors; (d) it is a stable monomer in the absence of an active site ligand; and (e) it interacts with the mammalian protein modulator pLG72 yielding a $\sim 100 \mathrm{kDa}$ complex in addition to the $\sim 200 \mathrm{kDa}$ one, as formed by the human DAAO. Furthermore, the concentration of endogenous D-serine in U87 glioblastoma cells was not affected by transfection with rat DAAO, whereas it was significantly decreased when expressing the human homologue. These results raise doubt on the use of the rat as a model system for testing new drugs against schizophrenia and indicate a different physiological function of DAAO in rodents and humans.

Structured digital abstract

- pLG72 binds rDAAO by molecular sieving (View interaction)

\section{Introduction}

D-Amino acid oxidase (DAAO; EC 1.4.3.3) is a FADcontaining flavoprotein that dehydrogenates the D-isomer of amino acids to the corresponding $\alpha$-keto acids and ammonia, coupled with the reduction of FAD. The cofactor then reoxidizes on molecular oxygen, yielding hydrogen peroxide [1]. In the human brain,

\footnotetext{
Abbreviations

CBIO, 6-chlorobenzo(d)isoxazol-3-ol; CPZ, chlorpromazine; DAAO, D-amino acid oxidase; EYFP, enhanced yellow fluorescent protein; hDAAO, human D-amino acid oxidase; NLS, N-lauroyl sarcosine; NMDAR, N-methyl-D-aspartate receptor; pkDAAO, pig kidney D-amino acid oxidase; rDAAO, rat D-amino acid oxidase.
} 
DAAO is involved in the degradation of D-serine, a neuromodulator acting as coagonist at the glycine site on the $N$-methyl-D-aspartate receptor (NMDAR). D-Serine is synthesized starting from its L-isomer by serine racemase (racemization reaction) and is metabolized by the $\alpha, \beta$-elimination reaction catalyzed by the same enzyme and/or by DAAO-catalyzed oxidative deamination [2]. Accordingly, human DAAO (hDAAO), expressed in different brain areas, might play a key role in regulating the activity of NMDAR: indeed, NMDAR hypofunction has been implicated in the pathophysiology of schizophrenia [2-4]. It has been proposed that, by increasing NMDA receptor function (e.g. by inhibiting D-serine degradation), schizophrenic symptoms might be ameliorated. This hypothesis is supported by various observations: D-serine levels in serum and cerebral spinal fluid are lower in schizophrenia patients than in healthy controls [5], whereas, in the same individuals, DAAO activity and expression levels are increased [6-8]. Furthermore, the oral administration of D-serine improved positive, negative and cognitive symptoms of schizophrenia patients as add-on therapy to typical and atypical antipsychotics [9]. The coadministration of D-serine with a DAAO inhibitor has been proposed as a more effective means of increasing the concentration of the neuromodulator in the brain [10].

This important physiological role of D-serine/DAAO coupling opened the way to the discovery of new drugs for schizophrenia treatment based on DAAO inhibition [10-13]: the rat has been widely used as an in vivo model for screening and testing such novel drugs. During the discovery of new hDAAO inhibitors by high-throughput screening, Duplantier et al. [13] reported that, for a number of compounds, rat DAAO (rDAAO) potencies were 50-100-fold weaker than for hDAAO; for example, for 3-hydroxyquinolin-2(1H)-one, $K_{\mathrm{i}}$ values were 424 and $9 \mathrm{~nm}$ for $\mathrm{rDAAO}$ and hDAAO, respectively. This observation has been proposed to explain the high free brain exposure required to increase in vivo D-serine levels in the rat [13] and prompted the same authors to choose the mouse instead of rat to evaluate the ability of the compounds (DAAO inhibitors) to increase cerebellar D-serine levels.

The nucleotide sequence of $\mathrm{rDAAO}$ cDNA was determined in 1998 [14]: it encodes for an enzyme of 346 amino acids, sharing $93 \%$ and $80 \%$ sequence identity with the mouse and human homologues, respectively. Noteworthy, all the active site residues are fully conserved between rat and mouse DAAOs. Among the 22 residues that differ between hDAAO and the rodent counterparts, seven are identical between human and rat, eight are identical between human and mouse, and only seven are different in all three enzymes: the sub- stitutions randomly distribute on the protein surface. A detailed biochemical and structural characterization of rDAAO, and a comparison with hDAAO, is crucial for endorsing the use of rat as an animal model for schizophrenia.

\section{Results}

\section{Biochemical properties of recombinant rDAAO}

Recombinant rDAAO was overexpressed in BL21 (DE3)Star Escherichia coli cells using the same conditions previously set up for hDAAO [15] and purified to $95 \%$ homogeneity by a single-step procedure on a metal-chelate affinity column (Fig. S1). A yield of rDAAO of $15 \mathrm{mg} \cdot \mathrm{L}^{-1}$ culture $\left(0.65 \mathrm{mg} \cdot \mathrm{g}^{-1} \cdot\right.$ cell $)$ was achieved.

The purified rDAAO shows the classical absorbance spectrum of FAD-containing flavoproteins, with maxima at 448, 368 and $274 \mathrm{~nm}$ (Fig. 1A, continuous line). The $A_{274} / A_{448}$ ratio, a parameter of protein purity, is $\sim 9.8\left(\varepsilon_{448 \mathrm{~nm}}=12.2 \pm 0.4 \mathrm{~mm}^{-1} \cdot \mathrm{cm}^{-1}\right)$. The oxidized form of the purified enzyme is immediately converted into the fully reduced species after adding the substrate D-alanine under anaerobic conditions (Fig. 1A, dashed line). Upon anaerobic photoreduction, oxidized rDAAO converts into the corresponding anionic semiquinone form: $\sim 90 \%$ of the one-electron reduced form of FAD was formed (Fig. 1A, dotted line). This species is kinetically stabilized because it is fully lost when mixed with benzyl viologen or when incubated in the dark overnight. The redox properties of rDAAO are provided in Appendix S1.

rDAAO shows good thermal stability: the melting temperature is $46.8 \pm 0.2{ }^{\circ} \mathrm{C}$ and $51.9 \pm 0.2{ }^{\circ} \mathrm{C}$ measuring the changes in protein and flavin fluorescence, respectively. These values also indicate that the loss of tertiary structure precedes FAD dissociation, as also reported for yeast DAAO [1].

Gel-permeation chromatography shows that rDAAO is a monomer in the concentration range $1-20 \mathrm{mg} \cdot \mathrm{mL}^{-1}$ for both the holoenzyme and the apoprotein forms (Fig. 2A), whereas hDAAO is a stable homodimer [16]. At 2, 10 and $20 \mathrm{mg} \cdot \mathrm{mL}^{-1}$ protein, the addition of $40 \mu \mathrm{M}$ FAD does not modify the elution profile of rDAAO holoenzyme, whereas, in the presence of $1 \mathrm{mM}$ benzoate a shift to an elution volume corresponding to an $\sim 80 \mathrm{kDa}$ homodimer is observed. These results indicate that there are significant alterations of the dimerization interface between the two mammalian DAAOs (see below). This is also apparent by monitoring the binding of the primate-specific modulator pLG72 protein by gel-permeation chromatography 


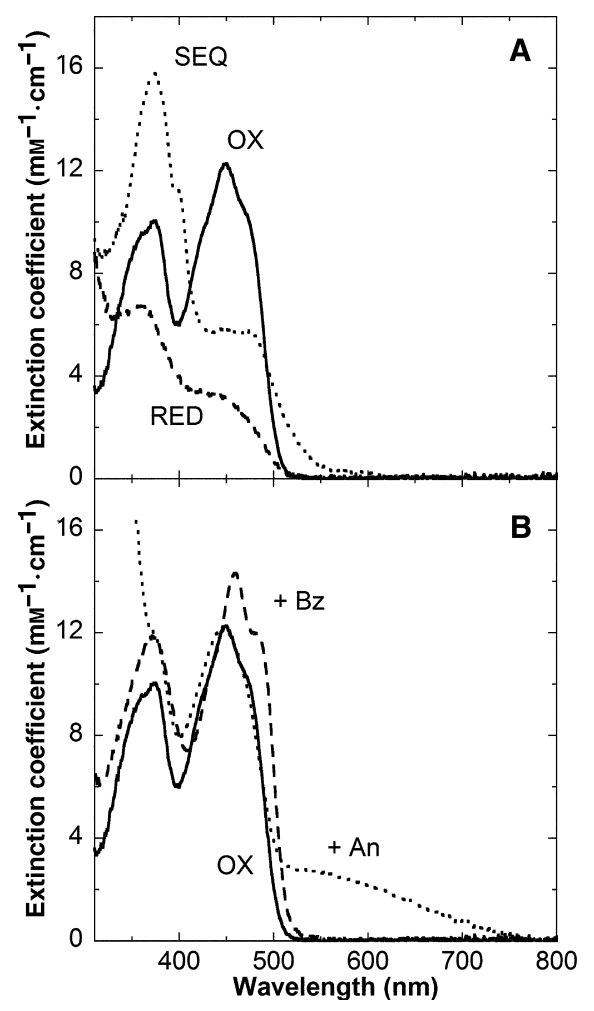

Fig. 1. Spectral properties of purified rDAAO. (A) Comparison of the visible absorbance spectra of oxidized ( - ), fully reduced (---; obtained by anaerobic addition of $310 \mathrm{~mm}$ D-alanine) and semiquinone form (...; obtained by light-induced anaerobic reduction) of rDAAO. (B) Perturbation of the visible spectrum of rDAAO after the addition of benzoate $(---; 1.3 \mathrm{~mm})$ and anthranilate $(. . ; 10 \mathrm{~mm})$. Spectra were recorded in $20 \mathrm{~mm}$ Tris- $\mathrm{HCl}(\mathrm{pH} 8.0), 10 \%$ glycerol and $5 \mathrm{~mm}$ 2-mercaptoethanol at $15{ }^{\circ} \mathrm{C}$, and using $\sim 0.4 \mathrm{mg} \cdot \mathrm{mL}^{-1}$ protein.

[17]. A mixture comprising a fixed amount of rDAAO $(25 \mathrm{nmol})$ and different amounts of pLG72 (12.5$100 \mathrm{nmol}$ ) was separated on a Superdex 200 column: the elution volume of rDAAO shifted from $\sim 16.1 \mathrm{~mL}$ to 14.2 and $12.8 \mathrm{~mL}$ (i.e. from the monomeric state of $40 \mathrm{kDa}$ to forms of $\sim 100$ and $200 \mathrm{kDa}$ ) (Fig. 2B). The presence of both pLG72 and hDAAO in these latter peaks was demonstrated by SDS/PAGE (Fig. 2B, inset). Furthermore, the relative intensity of the two peaks was similarly modified by altering the rDAAOpLG72 ratio and the $100 \mathrm{kDa}$ complex was also apparent when an excess of rDAAO was used. This observations exclude the $100 \mathrm{kDa}$ complex as a precursor of the bigger one and indicate two alternative modes of interaction of pLG72 with rDAAO. This behaviour differs from that observed for hDAAO where an $\sim 100 \mathrm{kDa}$ complex was never produced: for the human flavoenzyme, and both using the dimeric holoenzyme or the monomeric apoprotein, an $\sim 200 \mathrm{kDa}$

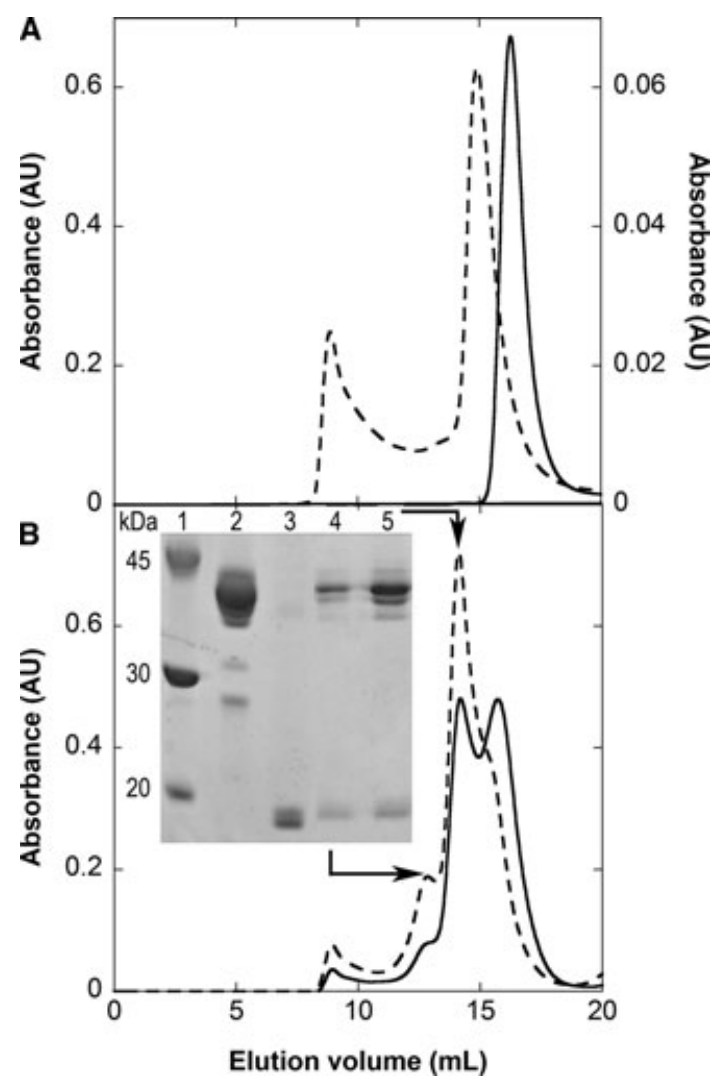

Fig. 2. Analysis of pLG72 binding to rDAAO by gel-permeation chromatography. Elution profiles of gel-permeation chromatography of: (A) $25 \mathrm{nmol}$ of purified rDAAO (—; left axis), and pLG72 (...; right axis) proteins; (B) mixtures containing $25 \mathrm{nmol}$ of rDAAO and different amounts of pLG72 (25 nmol, - , or $50 \mathrm{nmol},---)$. Peaks were analyzed by nonlinear curve fitting (using PEAKFIT software). (B, inset) SDS/PAGE analysis of purified rDAAO (5 $\mu \mathrm{g}$, lane 2) and pLG72 (5 $\mu \mathrm{g}$, lane 3) and of the rDAAO-pLG72 complexes isolated by gel-permeation chromatography (lane 4, peak at $12.8 \mathrm{~mL}$, $\sim 200 \mathrm{kDa}$ complex; lane 5, peak at $14.2 \mathrm{~mL}, \sim 100 \mathrm{kDa}$ complex). Lane 1, standard proteins.

complex comprising two pLG72 monomers and four hDAAO monomers was the only complex formed [17].

The model of rDAAO was built using SWISSMODEL and the 3D structure of hDAAO in complex with 3-hydroxyquinolin-2(1H)-one as the template [13]. As expected, there were no significant differences in the overall tertiary structure between the two mammalian DAAOs (Fig. 3A): a large part of the substitutions localize on the protein surface (Fig. 3B). Focusing on the monomer-monomer interface, three residues distinguish rDAAO (as well as the mouse counterpart) from hDAAO: (a) R120 in hDAAO, involved in electrostatic interactions with the carboxylic group of D73 and hydrogen-bonded to the backbone $\mathrm{C}=\mathrm{O}$ groups of D109, T110 and V111, is replaced by S119 in rDAAO; (b) the residue hDAAO-Q234 in rDAAO is 


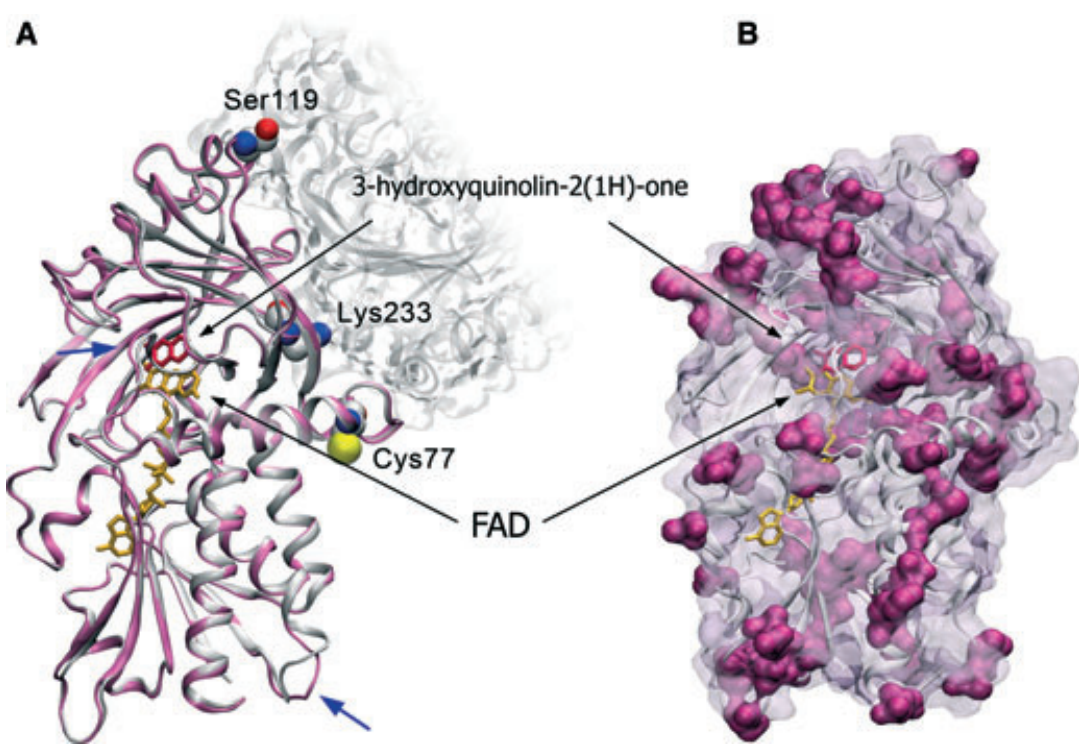

Fig. 3. 3D model of rDAAO. (A) Structural overlay of the model of tertiary structure of rDAAO (purple) with the structure of hDAAO (gray, Protein Data Bank code 3g3e): major differences in loop orientations are indicated by blue arrows and three residues of rDAAO not conserved in hDAAO (and belonging to its monomer-monomer surface) are also indicated. (B) Surface representation of rDAAO, highlighting (in purple) the residues that are not conserved in the human counterpart.

substituted by $\mathrm{K} 233$; and (c) hDAAO-H78 is replaced by $\mathrm{C} 77$ in rDAAO.

\section{Steady-state kinetics}

Apparent steady-state kinetic parameters of rDAAO were determined at air saturation using the oxygenconsumption assay on different D-amino acids and glycine as substrates. As shown in Table 1, rDAAO shows the highest affinity for bulky and hydrophobic substrates, such as D-tryptophan, D-phenylalanine and D-proline, compared to substrates with small side chains. Glycine is not a substrate of rDAAO (no activity was detected up to $1 \mathrm{M}$ ), as well as D-aspartate (no activity up to $0.5 \mathrm{M}$ ). rDAAO substrate preferences differ significantly from those of hDAAO (Table 1) [16]: the $K_{\mathrm{m} \text {,app }}$ for D-alanine and D-serine is $\sim 100$ and 40-fold higher with respect to the human enzyme, whereas $k_{\text {cat,app }}$ values are five- and two-fold higher. The highest kinetic efficiency $\left(k_{\text {cat,app }} / K_{\text {m,app }}\right)$ determined for rDAAO is one order of magnitude lower than for hDAAO or pig kidney DAAO (pkDAAO) and three orders of magnitude lower than for the yeast enzyme $[1,18]$.

The $\mathrm{O}_{2}$-dependence of the reaction of rDAAO was investigated by the enzyme monitored turnover method by rapidly mixing the oxidized enzyme aerobically with D-alanine (concentration range $1.3-10 \mathrm{mM}$ ) and monitoring the time course of spectral changes (data not shown). The absorbance traces at $455 \mathrm{~nm}$ were analyzed as a function of oxygen concentration as described previously [18]. Lineweaver-Burk plots at different substrate concentrations show a set of parallel lines (not shown). The same behaviour was reported previously for pkDAAOs and hDAAOs on neutral substrates $[16,19]$ and, by a combination of steadystate and pre-steady-state measurements, it was demonstrated to arise from a ternary complex kinetic mechanism with some of the rate constants sufficiently small such that the bimolecular term of the steadystate equation becomes negligible at high substrate concentrations (Table 2). The steady-state coefficients confirm the low affinity of rDAAO for D-alanine, as obtained at a fixed oxygen concentration (see above), whereas the affinity for dioxygen resembles that of known mammalian DAAOs (Table 2).

\section{FAD binding}

The apoprotein form of rDAAO was prepared by a dialysis procedure based on the use of a high concentration of a chaotropic salt $(2 \mathrm{M} \mathrm{KBr})$, with a final yield of $\sim 24 \%$ in terms of protein recovered. After incubation of the apoprotein with a ten-fold molar excess of $\mathrm{FAD}$, the reconstituted holoenzyme recovered $\sim 30 \%$ of the specific activity of native rDAAO. The rDAAO apoprotein maintains the monomeric state (see above) and the secondary structure content 
Table 1. Comparison of substrate specificity of rDAAO and hDAAO. The apparent kinetic parameters were determined using the oxygenconsumption assay at $25^{\circ} \mathrm{C}$ and $\mathrm{pH}$ 8.3. $\mathrm{BD}$, below detection limits.

\begin{tabular}{|c|c|c|c|c|c|c|}
\hline \multirow[b]{2}{*}{ Substrate } & \multicolumn{3}{|l|}{ rDAAO } & \multicolumn{3}{|l|}{ hDAAO ${ }^{a}$} \\
\hline & $k_{\text {cat, app }}\left(\mathrm{s}^{-1}\right)$ & $K_{\mathrm{m}, \text { app }}(\mathrm{mM})$ & $k_{\mathrm{cat}} / K_{\mathrm{m}}\left(\mathrm{mm}^{-1} \cdot \mathrm{s}^{-1}\right)$ & $k_{\text {cat, app }}\left(\mathrm{s}^{-1}\right)$ & $K_{\mathrm{m}, \text { app }}(\mathrm{mM})$ & $k_{\text {cat }} / K_{\mathrm{m}}\left(\mathrm{mm}^{-1} \cdot \mathrm{s}^{-1}\right)$ \\
\hline D-Ala & $27 \pm 1$ & $\begin{aligned} & 140 \pm 20 \\
\sim & 100^{\mathrm{b}} \\
& 102 \pm 32^{\mathrm{c}}\end{aligned}$ & 0.20 & $5.2 \pm 0.1$ & $1.3 \pm 0.2$ & 4.0 \\
\hline D-Ser & $6.4 \pm 0.8$ & $310 \pm 60$ & 0.02 & $3.0 \pm 0.1$ & $7.5 \pm 0.5$ & 0.4 \\
\hline D-Pro & $47 \pm 1$ & $\begin{aligned} & 86 \pm 22 \\
\sim & 31^{\mathrm{b}} ; \sim 4.2^{\mathrm{d}} \\
& 68 \pm 14^{\mathrm{c}}\end{aligned}$ & 0.55 & $10.2 \pm 0.1$ & $8.5 \pm 10$ & 1.2 \\
\hline D-Trp & $3.7 \pm 0.2$ & $15 \pm 1$ & 0.25 & $3.2 \pm 0.1$ & $1.5 \pm 0.1$ & 2.1 \\
\hline D-Phe & $8.5 \pm 0.2$ & $35 \pm 9$ & 0.24 & $6.6 \pm 0.1$ & $2.7 \pm 0.2$ & 2.4 \\
\hline Gly & $\mathrm{BD}$ & & & $\sim 0.9$ & $\sim 180$ & 0.005 \\
\hline
\end{tabular}

a Molla et al. [16]. ${ }^{b}$ Huynh et al. [30]. ${ }^{c}$ Rat kidney extract. ${ }^{d}$ Determined from cytophotometric analysis of pig liver sections [38].

Table 2. Comparison of steady-state coefficients of rDAAO and hDAAO determined by rapid mixing techniques [18] at $\mathrm{pH} 8.3$ and $25^{\circ} \mathrm{C}$. The steady-state parameters have been determined using the equation for a ternary complex mechanism [18,19,33]: $\mathrm{e}_{\mathrm{t}} / \mathrm{V}=\Phi_{0}+\Phi_{\mathrm{D}-\mathrm{Ala}} /[\mathrm{D}-\mathrm{Ala}]+\Phi_{\mathrm{O}_{2}} /\left[\mathrm{O}_{2}\right]+\Phi_{\mathrm{D}-\mathrm{Ala}, \mathrm{O}_{2}} /[\mathrm{D}-\mathrm{Ala}]\left[\mathrm{O}_{2}\right]$, where $k_{\text {cat }}=1 / \Phi_{0} ; K_{\mathrm{m}, \mathrm{D}-\mathrm{Ala}}=\Phi_{\mathrm{D}-\mathrm{Ala}} / \Phi_{0} ; K_{\mathrm{m}, \mathrm{O}_{2}}=\Phi_{\mathrm{O}_{2}} / \Phi_{0} ; \Phi_{\mathrm{D}-\mathrm{Ala}, \mathrm{O}_{2}} \sim 0$.

\begin{tabular}{lll}
\hline & rDAAO & hDAAO $^{a}$ \\
\hline Lineweaver-Burk plot & Parallel & Parallel \\
$K_{\text {cat }}\left(\mathrm{s}^{-1}\right)$ & $40 \pm 10$ & $14.7 \pm 0.7$ \\
$K_{\mathrm{m}, \mathrm{D}-\mathrm{Ala}}(\mathrm{mm})$ & $\geq 100$ & $8.8 \pm 0.3$ \\
$K_{\mathrm{m}, \mathrm{O}_{2}}(\mathrm{mM})$ & $0.5 \pm 0.1$ & $1.2 \pm 0.3$ \\
$\Phi_{\mathrm{D}-\mathrm{Ala}}\left(\mathrm{M} \mathrm{s} \times 10^{-3}\right)$ & $3.3 \pm 1.0$ & $0.6 \pm 0.1$ \\
$\Phi_{\mathrm{O}_{2}}\left(\mathrm{M} \mathrm{s} \times 10^{-5}\right)$ & $1.3 \pm 0.2$ & $8 \pm 0.3$ \\
\hline
\end{tabular}

${ }^{a}$ Molla et al. [16].

of the holoenzyme (i.e. their far-UV CD spectra are similar). Instead, significant changes are apparent in the near-UV CD spectrum: the holoenzyme shows a negative peak at $\sim 270 \mathrm{~nm}$ that is absent in the apoprotein (data not shown). These structural properties of rDAAO apoprotein closely resemble those of human and yeast DAAOs [16,20,21].

The $K_{\mathrm{d}}$ for FAD binding to rDAAO apoprotein was determined by measuring the quenching of protein fluorescence after adding increasing amounts of the cofactor: a value of $3.0 \pm 0.8 \times 10^{-7} \mathrm{M}$ was calculated. This value is similar to that determined for pkDAAO $\left(2 \times 10^{-7} \mathrm{M}\right)$ and $\sim 25$-fold tighter than the FAD binding to hDAAO (Table 3). Interestingly, in the presence of benzoate, the $K_{\mathrm{d}}$ for FAD binding to hDAAO apoprotein is significantly lower $(3 \pm 1 \times$ $\left.10^{-7} \mathrm{M}\right)$ : a similar effect of ligand binding on the cofactor interaction is not apparent for rDAAO (Table 3).
Table 3. Comparison of binding properties of rDAAO and hDAAO. $K_{d}$ values for $F A D$ and $C P Z$ binding to rDAAO apoprotein were determined by monitoring the quenching of protein fluorescence; $K_{d}$ values for inhibitors binding to rDAAO holoenzyme were determined spectrophotometrically by monitoring the perturbation of flavin absorbance spectrum (for details, see Figs 1 and 4). The $K_{\mathrm{i}}$ value determined by the oxygen-consumption assay on $\mathrm{D}$-alanine as substrate is shown in square parenthesis.

\begin{tabular}{lll}
\hline & \multicolumn{2}{l}{$K_{\mathrm{d}}$} \\
\cline { 2 - 3 } & rDAAO & hDAAO \\
\hline FAD binding (M) & $(3.0 \pm 0.8) \times 10^{-7}$ & $(8.0 \pm 2) \times 10^{-6 a}$ \\
FAD & $(3.8 \pm 0.3) \times 10^{-7}$ & $(3.0 \pm 1) \times 10^{-7 a}$ \\
FAD + benzoate & & \\
& & \\
Inhibitors binding (mM) & & $0.007 \pm 0.002^{\mathrm{a}}$ \\
Benzoate & $0.15 \pm 0.01 ; \sim 0.10^{\mathrm{b}}$ & \\
& {$\left[K_{\mathrm{i}}=0.40 \pm 0.07\right]$} & $0.04 \pm 0.01^{\mathrm{a}}$ \\
Anthranilate & $5.5 \pm 1.2$ & \\
& {$\left[K_{\mathrm{i}}=1.6 \pm 0.2\right]$} & $0.26 \pm 0.04$ \\
Crotonate & $2.9 \pm 0.2$ & $0.101 \pm 0.024$ \\
CBIO & $0.075 \pm 0.007$ & $0.005 \pm 0.0001^{\mathrm{c}}$ \\
CPZ & $0.009 \pm 0.001$ & $0.0064 \pm 0.009^{\mathrm{a}}$ \\
Sulfite & $220 \pm 48$ & \\
\hline
\end{tabular}

${ }^{a}$ Molla et al. [16]. ${ }^{b}$ Huynh et al. [30]. ${ }^{c}$ Sacchi et al. [17]; Caldinelli et al. [23].

\section{Inhibitor binding}

The $K_{\mathrm{d}}$ values for binding of carboxylic acids benzoate, crotonate and anthranilate (well-known DAAO inhibitors) [1] to rDAAO holoenzyme were determined spectrophotometrically by titrating a fixed amount of rDAAO with increasing concentrations of each compound. Binding of these inhibitors yields classical 


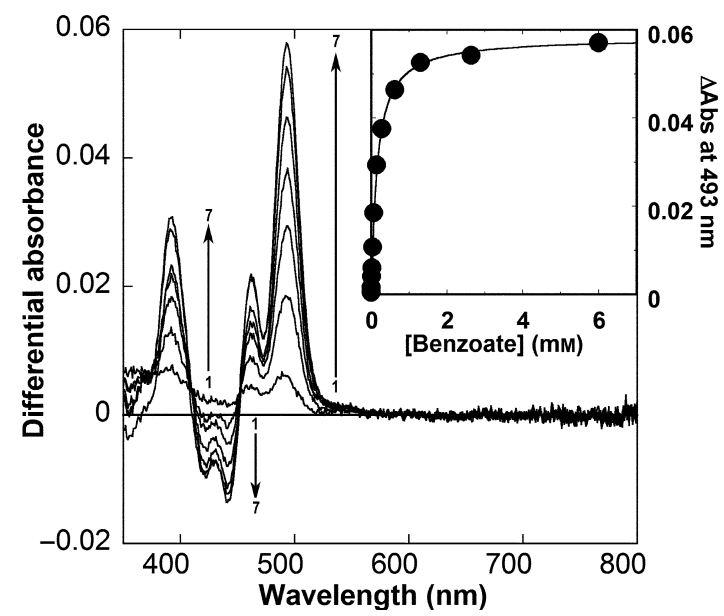

Fig. 4. Difference spectra for binding of benzoate to rDAAO. The difference spectra were obtained by subtracting the absorbance spectrum of the free oxidized form of rDAAO $(10.6 \mu \mathrm{M})$ from the spectrum of the same enzyme after adding $0.019,0.0725,0.14$, $0.28,0.62,2.64$ and $12.7 \mathrm{~mm}$ sodium benzoate (curves 1-7; see the versus of the arrows), at $15^{\circ} \mathrm{C}$ (Fig. 1B). Inset: plot of change in $A_{493}$ as a function of benzoate concentration; continuous line: fit based on a hyperbolic equation: $\Delta \mathrm{Abs}=\Delta \mathrm{Abs}_{\text {tot }} \times$ [benzoate] $/$ $\left(K_{\mathrm{d}}+\right.$ [benzoate]).

perturbations of the absorption spectrum of the enzyme in the visible region (Fig. 1B), as previously observed for other mammalian and yeast DAAOs [1]. The absorbance changes (at $493 \mathrm{~nm}$ for benzoate and crotonate and at $550 \mathrm{~nm}$ for anthranilate) at increasing concentrations of ligands show a classical saturation behaviour and the data were fit using a hyperbolic equation (for benzoate, see Fig. 4). A weaker interaction for the inhibitors tested is apparent for rDAAO compared to the mammalian counterparts: $K_{\mathrm{d}}$ values for benzoate and anthranilate are 20- and 130-fold higher, respectively, than the values determined for the human homologue (Table 3).

The oxygen-consumption assay at increasing D-alanine concentrations and in the presence of different concentrations of benzoate $(0-2 \mathrm{~mm})$ or anthranilate $(0-15 \mathrm{mM})$ was used to assess the mechanism of rDAAO inhibition. Both compounds are competitive versus the substrate because the linear fit of the experimental data obtained at different D-alanine concentrations and fixed inhibitor concentrations in the $1 / v$ versus $1 /[\mathrm{D}-\mathrm{Ala}]$ double reciprocal plot converge on the $y$-axis (for benzoate, see Fig. S2). The $K_{\mathrm{i}}$ values resemble the $K_{\mathrm{d}}$ values obtained spectrophotometrically (Table 3 ).

We also investigated the binding of 6-chlorobenzo(d)isoxazol-3-ol (CBIO), which belongs to the newly identified group of hDAAO inhibitors [10], by measuring the perturbation of the flavin absorbance spectrum: $K_{\mathrm{d}}$ is $75 \pm 7 \mu \mathrm{M}$. Interestingly, and in contrast to the classical carboxylic acid DAAO inhibitors (see above), this compound binds rDAAO similarly to hDAAO $\left(K_{\mathrm{d}}=101 \pm 24 \mu \mathrm{M}\right)$. Indeed, the $K_{\mathrm{d}}$ value strictly resembles the $\mathrm{IC}_{50}$ value determined previously $(189 \mu \mathrm{M})$ [10].

The drug chlorpromazine (CPZ), which is an aliphatic phenothiazine and a widely used antipsychotic drug in the treatment of schizophrenia, possesses a chemical structure resembling that of the isoalloxazine ring of FAD. CPZ is known as an FAD competitive inhibitor of pkDAAO [22] and interacts with the apoprotein form of hDAAO, as well as with its putative modulator pLG72, with a $K_{\mathrm{d}}$ of $\sim 5 \mu \mathrm{M}$ [17]. The strength of the rDAAO-CPZ interaction, as determined following the quenching of the protein fluorescence of rDAAO apoprotein at increasing CPZ concentrations, is similar to that determined for the human counterpart: a $K_{\mathrm{d}}$ of $9.9 \pm 1.0 \mu \mathrm{M}$ was estimated (Table 3 ).

\section{Properties of rDAAO from rat tissues}

To rule out the possibility that the characterized features of rDAAO arise from its recombinant origin and thus differ from those of the native enzyme, the flavoenzyme was isolated (and partially purified) from rat kidneys. After ammonium sulfate precipitation, the specific activity of the protein preparation was $\sim 67 \mathrm{mU} \cdot \mathrm{mg}^{-1}$ protein, indicating that rDAAO corresponds to $0.20 \%$ of the overall protein content (a value of $0.27 \%$ was obtained by western blot analysis of the same sample). This preparation was used to assay the kinetics on a number of compounds: $K_{\mathrm{m}}$ values were $110 \pm 30$ and $68 \pm 14 \mathrm{~mm}$ for D-alanine and D-proline, respectively (compare with values given in Table 1 for recombinant rDAAO). This preparation was also inactive on D-aspartate and D-glutamate (final concentration $0.5 \mathrm{M}$ ), thus confirming the absence of detectable amounts of D-aspartate oxidase. Benzoate was demonstrated also to be a competitive inhibitor of native rDAAO, with a $K_{\mathrm{i}}$ of $0.57 \mathrm{~mm}$ versus D-alanine as substrate (Fig. S3 and Table 3). This minimal characterization shows that the main kinetic and binding properties of rDAAO purified from rat kidney strictly resemble those of the recombinant enzyme (see above).

When the same procedure was used to isolate rDAAO from rat brain, no DAAO-specific signal was apparent by western blot analysis (loading up to $70 \mu \mathrm{g}$ of proteins; not shown), nor was any enzymatic activity detected in the crude extract. This result highlights a very low expression of rDAAO in the brain, as also suggested previously [14]. 


\section{Effect of rDAAO on cellular D-serine concentration}

To investigate the effect of rDAAO on the cellular level of D-serine, we produced stably transfected U87 human glioblastoma cells expressing enhanced yellow fluorescent protein (EYFP)-rDAAO. The expression of the chimeric protein was demonstrated by fluorescence microscopy, western blot analysis (Fig. 5A) and an activity assay using the Amplex UltraRed method (46 $\pm 8 \mathrm{mU} \cdot \mathrm{mg}^{-1}$ protein). The $\mathrm{D}$ - and L-serine concentrations were determined by HPLC chromatography. Although U87 cells transfected with hDAAO showed a statistically significant decrease in the $\mathrm{D} /(\mathrm{D}+\mathrm{L})$ serine concentration ratio compared to controls [17,23], the overexpression of EYFP-rDAAO did not affect this value to a statistically significant extent (Fig. 5B).

\section{Discussion}

In 2005, a trial in the USA comprising almost 1500 patients, which aimed to test a group of anti-psychotic drugs introduced in the $1990 \mathrm{~s}$, demonstrated that these drugs were no better than perphenazine, a 50-year-old drug that caused serious side effects. Thus, it was confirmed that the new drugs were almost the same as the old ones [24]. Typical antipsychotics exert their effect by blocking the dopamine type 2 receptor, thus affecting dopamine neurotransmission. In clinical trials, several candidate drugs are being tested that act on receptors related to the neurotransmitter glutamate. It is importance to note that animal tests (i.e. of memory, attention and other aspects of cognition) are largely employed to test drug candidates: unreliable tests may explain why drug candidates who appear hopeful in animal models fail in clinical use. Indeed, novel drugs to treat schizophrenia by acting on DAAO (and hence aim to alter D-serine metabolism) have been tested on rats $[10,11,13,25]$; therefore, we decided to investigate the properties of rDAAO.

Previous studies on rDAAO focused on its distribution not only specifically in the central nervous system [26], but also in the peripheral nervous system [27], as well as its cellular and subcellular distribution [26,28]. Most recently, LEA/SENDAI rats lacking DAAO activity (i.e. no DAAO mRNA was produced) have been identified [29]. To date, the only available biochemical data on rDAAO are related to a partially purified enzyme from kidney [30]: with this enzyme preparation, a $K_{\mathrm{m} \text {,app }}$ value for D-alanine and a $K_{\mathrm{i}}$ for benzoate similar to those reported in the present study for recombinant $\mathrm{rDAAO}$ were determined (Tables 1 and 3). Analogously, our basic characterization of
A
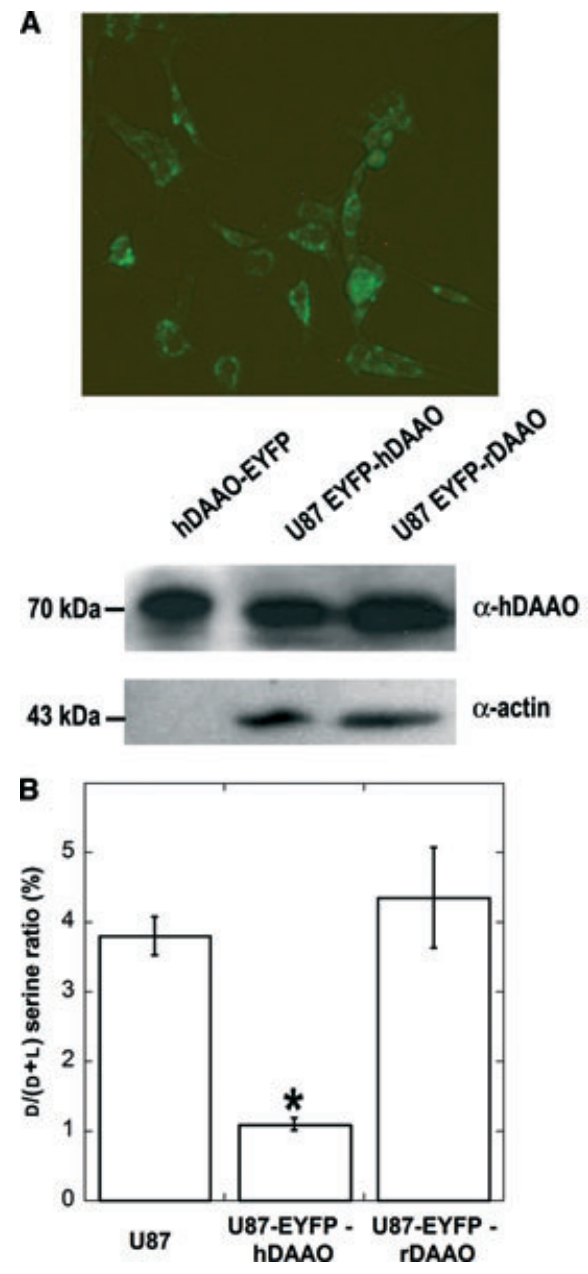

Fig. 5. Dependence of D-serine concentration on DAAO stable transfection of U87 glioblastoma cells. (A) Top: analysis of expression of chimeric fluorescent EYFP-rDAAO protein in stably transfected U87 glioblastoma cells. The image was acquired by a fluorescence microscope and shows the level and distribution of the expressed chimeric protein. Bottom: western blot analysis performed using rabbit anti-hDAAO sera confirms the expression of EYFP-hDAAO or EYFP-rDAAO fluorescent fusion proteins in transfected U87 cells. Approximately $5 \times 10^{4}$ cells were loaded in each lane; as an internal control, the amount of loaded protein was assessed using anti- $\alpha$-actin serum (bottom panel). EYFP-hDAAO $(0.03 \mu \mathrm{g}$, left lane) recombinant purified protein was used as a positive control. (B) The histogram reports the $D /(D+L)$ serine concentration ratio in U87 cells stably transfected with EYFP-rDAAO or EYFP-hDAAO compared to control cells. The values are expressed as a percentage. The change in the $D /(D+L)$ serine ratio was found not to be significant for rDAAO versus control $(P=0.59)$ and significant for hDAAO $\left({ }^{*} P<0.00015\right)$. The data are reported as the mean $\pm \mathrm{SE}$; for each point, at least six independent determinations were performed.

rDAAO isolated from rat kidney also confirms that the properties of the recombinant enzyme resemble those of the native protein. 
The biochemical characterization of rDAAO shows that it differs significantly from the human enzyme, particularly with regard to its binding properties. Importantly, a comparison of the hDAAO structure with the model of $\mathrm{PDAAO}$ shows that all residues at $<8 \AA$ from the ligand are conserved in the two enzymes. A comparison of the active site of human and pig DAAOs with the model obtained for rDAAO is depicted in Fig. S4. The hDAAO protein weakly binds FAD, and this interaction is made ten-fold stronger by ligand binding [16], reaching values similar to those for uncomplexed rDAAO and pkDAAO (Table 3). The most significant alterations are apparent with regard to substrate affinity: $K_{\mathrm{m}}$ and the kinetic efficiency for D-serine are 40- and 20-fold lower, respectively, for $\mathrm{rDAAO}$ than for hDAAO (Table 1). rDAAO is the worst catalyst among the known DAAOs [1]: for all tested substrates, the kinetic efficiency is at least one order of magnitude lower than for other mammalian DAAOs (Table 1), whereas $K_{\mathrm{m}, \mathrm{O}_{2}}$ is only marginally different (Table 2). This difference explains the lack of an effect of rDAAO transfection in U87 cells on the cellular D-serine concentration (Fig. 5B). Concerning the binding of carboxylic acids, binding to rDAAO is $50-150$-fold weaker than to the human (and porcine) counterpart for all the compounds tested, with the sole exception of the largest one (i.e. CBIO), for which a similar affinity is apparent between rDAAO and hDAAO (Table 3). This result confirms the preference of $\mathrm{rDAAO}$ for the binding of bulky, hydrophobic compounds (i.e. the lowest $K_{\mathrm{m}}$ is observed for D-tryptophan) (Table 1), and suggests that only large, aromatic carboxylic acids could efficiently inhibit both rat and human DAAOs. Furthermore, the oligomeric state of rDAAO holoenzyme is different from that of hDAAO and pkDAAOs: in solution, free rDAAO is always present as a stable monomer and converts into a homodimer after the binding of benzoate. An inspection of monomer-monomer interaction regions between $\mathrm{hDAAO}$ and the model for rDAAO (Fig. 3) indicates that the substitution of R120 in hDAAO with S119 in rDAAO might be mainly responsible for the weaker interaction between monomers in the latter. Moreover, the interaction of rDAAO with the modulator pLG72 also differs from hDAAO [17]: an $\sim 100 \mathrm{kDa}$ ensuing complex (most likely comprising two rDAAO monomers and one pLG72 monomer) was never observed with the human homologue. This is an intriguing result because yeast DAAO (which shows a different mode of monomermonomer interaction compared to mammalian DAAOs; i.e. head-to-tail versus head-to-head, respectively) [1] does not interact with pLG72 [17]. This observation suggests that the primate-specific pLG72 protein specifically evolved to interact with human DAAO.

In conclusion, the different properties of $\mathrm{rDAAO}$ compared to hDAAO explain the observation that the acute inhibition of rDAAO does not appear to be sufficient to increase D-serine concentrations to the levels required to produce an antipsychotic and cognitionenhancing effect in rats (i.e. as is observed after administration of high doses of exogenous D-serine) [25] and suggest that DAAO has a different physiological function in rodents and humans and/or that different catabolic pathways for D-serine exist. From a pharmacological point of view, the results obtained in the present study raise doubts regarding the use of rat as a model system for testing new drugs to treat schizophrenia and highlight the danger of applying noncritically to humans the intriguing observation that, in rats, DAAO might represent a target molecule for the treatment of chronic pain of neuropathic origin [31].

\section{Experimental procedures}

\section{Design, synthesis and cloning of cDNA encoding for rDAAO}

Plasmid DNA was extracted and purified using NucleoSpin $^{\circledR}$ Extract kit (Macherey-Nagel, Düren, Germany). cDNA coding for rDAAO was a generous gift from Larry Melnick (Sunovion Pharmaceuticals, Marlborough, MA, USA). This cDNA was cloned into the expression plasmid pET28 vector, carrying kanamicin resistance, using restriction sites for $N d e$ I and $S a l$ (pET28-rDAAO): by applying this cloning strategy, rDAAO can be expressed with a N-terminal His-tag.

\section{Expression and purification of recombinant rDAAO}

Recombinant rDAAO was expressed in the BL21(DE3)Star E. coli strain, which is the best host for expressing hDAAO [15]. Starter cultures were prepared, growing a single colony of $E$. coli cells carrying the recombinant plasmid overnight at $37^{\circ} \mathrm{C}$ in flasks containing Terrific broth (Sigma-Aldrich, St Louis, MO, USA) to which $30 \mu \mathrm{g} \cdot \mathrm{mL}^{-1}$ kanamicin was added; this culture was diluted in the same medium to a starting value of 0.025 at $D_{600}$. For preparative purposes, $2 \mathrm{~L}$ baffled Erlenmeyer flasks containing $200 \mathrm{~mL}$ of liquid Terrific broth medium were used. Cells were grown at $37{ }^{\circ} \mathrm{C}$ with shaking (200 r.p.m.) up to the stationary phase, when $0.6 \mathrm{~mm}$ isopropyl thio- $\beta$-D-galactoside was added; cells were collected after $2 \mathrm{~h}$.

Cell pellets were resuspended in freshly prepared lysis buffer ( $3 \mathrm{~mL} \cdot \mathrm{g}^{-1}$ wet cells) containing $50 \mathrm{~mm}$ sodium pyrophosphate $\left(\mathrm{pH} 8.3\right.$ ), 5\% glycerol, $0.7 \mu \mathrm{g} \cdot \mathrm{mL}^{-1}$ pepstatin, $40 \mu \mathrm{M}$ 
FAD, $5 \mathrm{~mm}$ 2-mercaptoethanol, $1.1 \mathrm{~mm}$ phenylmethylsulfonyl fluoride and $10 \mu \mathrm{g} \cdot \mathrm{mL}^{-1}$ deoxyribonuclease I and disrupted by sonication (six cycles of $30 \mathrm{~s}$ each, on ice, using a Branson Sonifier 250; Branson Ultrasonics, Danbury, CT, USA). The insoluble fraction was removed by centrifugation at $39000 \mathrm{~g}$ for $1 \mathrm{~h}$ at $4{ }^{\circ} \mathrm{C}$. Recombinant rDAAO was purified up to $95 \%$ homogeneity (estimated by SDS/PAGE) by a single-step procedure on a $\mathrm{Ni}^{2+}$-chelate affinity column (HiTrap Chelating; GE Healthcare, Piscataway, NJ, USA), equilibrated in $50 \mathrm{~mm}$ sodium pyrophosphate ( $\mathrm{pH} 7.2), 1 \mathrm{M}$ $\mathrm{NaCl}$ and $5 \mathrm{~mm}$ 2-mercaptoethanol. A first step at $5 \%$ of elution buffer $[50 \mathrm{~mm}$ sodium pyrophosphate $(\mathrm{pH}$ 7.2), $0.5 \mathrm{~m}$ imidazole, $5 \%$ glycerol and $5 \mathrm{~mm} 2$-mercaptoethanol] eliminated contaminant proteins weakly bound to the column. Then, the bound enzyme was eluted at $100 \%$ of elution buffer. The purified rDAAO was equilibrated in storage buffer [20 mM Tris- $\mathrm{HCl}, \mathrm{pH} 8.0,0.1 \mathrm{M} \mathrm{NaCl}, 10 \%$ glycerol (v/v) and $5 \mathrm{~mm}$ 2-mercaptoethanol] by gel-permeation chromatography on a PD10 column (GE Healthcare) and stored at $-20{ }^{\circ} \mathrm{C}$ : no loss of enzymatic activity was observed up to 6 months.

The apoprotein form of rDAAO was produced by extensive dialysis $(\sim 40 \mathrm{~h})$ of $2 \mathrm{mg} \cdot \mathrm{mL}^{-1}$ rDAAO against $50 \mathrm{~mm}$ sodium pyrophosphate $(\mathrm{pH} 8.3), 2 \mathrm{M}$ potassium bromide and $1 \mathrm{~mm}$ EDTA. Apoprotein was then equilibrated in $50 \mathrm{~mm}$ sodium pyrophosphate, $10 \%$ glycerol (v/v) and $5 \mathrm{~mm}$ 2-mercaptoethanol.

\section{Kinetic measurements}

Steady-state kinetic measurements on different D-amino acids as substrate were performed in $75 \mathrm{~mm}$ sodium pyrophosphate buffer ( $\mathrm{pH} \mathrm{8.5)}$ at $25^{\circ} \mathrm{C}$ and air saturation $(0.253 \mathrm{~mm})$, measuring the oxygen consumption with a Hansatech (King's Lynn, UK) oxygen electrode [16]. One rDAAO unit corresponds to the amount of enzyme that converts $1 \mu \mathrm{mol} \cdot \mathrm{min}^{-1}$ D-amino acid or oxygen. The effect of various compounds on rDAAO activity on D-alanine as substrate was determined by the same assay, measuring the initial reaction rate in assay mixtures containing different concentrations of inhibitor and substrate [32]. The initial reaction rates were used to calculate the kinetic parameters with KALEIDAGRAPH software (Synergy Software, Reading, PA, USA).

The rapid mixing experiments were performed in $50 \mathrm{~mm}$ sodium pyrophosphate buffer $(\mathrm{pH} 8.3)$, containing 10\% glycerol, at $25^{\circ} \mathrm{C}$ in a stopped-flow BioLogic (Grenoble, France) SFM-300 spectrophotometer equipped with a J\&M diode array detector (J\&M Analytische Mess-und Regeltechnik GmbH, Aalen, Germany) [33]. Enzyme-monitored turnover data were analyzed as described previously [17].

\section{Spectroscopic experiments}

All spectroscopic experiments were performed at $15^{\circ} \mathrm{C}$ in $20 \mathrm{~mm}$ Tris- $\mathrm{HCl}(\mathrm{pH} 8.0), 10 \%$ glycerol and $5 \mathrm{~mm}$ 2-mercaptoethanol. Dissociation constants for ligands were determined spectrophotometrically by adding small volumes $(1-10 \mu \mathrm{L})$ of concentrated stock solutions of ligands to samples containing $1 \mathrm{~mL}$ of $\sim 10 \mu \mathrm{M}$ enzyme and calculated from the change in $A_{493}$ for benzoate and crotonate, $A_{550}$ for anthranilate and $A_{450}$ for sodium sulfite [16,34].

$\mathrm{O}_{2}$-free samples were prepared in anaerobic cuvettes by applying ten cycles of evacuation/flushing with oxygen-free argon [16]. Photoreduction experiments were carried out in an anaerobic cuvette containing $10 \mu \mathrm{M}$ enzyme in the presence of EDTA and 5-deaza-riboflavin as described previously [34]. The redox potentials for the oxidized/reduced rDAAO couple were determined by employing the dye equilibration method described previously [35] at $15^{\circ} \mathrm{C}$. The enzyme solution was mixed in an anaerobic cuvette with $0.2 \mathrm{~mm}$ xanthine, $5 \mu \mathrm{M}$ benzyl viologen as mediator and $40 \mu \mathrm{M}$ of 2-hydroxy-1,4-naphthoquinone as reference dye: the reaction was initiated by adding $10 \mathrm{~nm}$ xanthine oxidase. Data were analyzed as described previously $[35,36]$.

All fluorescence measurements were performed at a protein concentration of $0.1 \mathrm{mg} \cdot \mathrm{mL}^{-1}$. The binding constant for FAD was determined by titrating $1 \mu \mathrm{M}$ apoprotein with increasing amounts of cofactor and by monitoring reconstitution measuring the quenching of protein fluorescence at $340 \mathrm{~nm}$ (excitation at $280 \mathrm{~nm}$ ). Temperature-ramp fluorescence experiments were performed using a software-driven, Peltier-based temperature controller (temperature gradient of $\left.0.5^{\circ} \mathrm{C} \cdot \mathrm{min}^{-1}\right)[16,20]$.

CD spectra were recorded on a Jasco J-810 spectropolarimeter and analyzed using JASCO software (Jasco Europe, Cremella, Italy). For measurements above $250 \mathrm{~nm}$, the cell path was $1 \mathrm{~cm}$ and, for measurements in the range 190$250 \mathrm{~nm}$, it was $0.1 \mathrm{~cm} \mathrm{[16].}$

\section{Oligomeric state, pLG72 binding and limited proteolysis}

The oligomeric state of rDAAO (free and complexed with pLG72) was determined by gel-permeation chromatography on a Superdex 200 column (GE Healthcare) in $20 \mathrm{~mm}$ Tris- $\mathrm{HCl}(\mathrm{pH} 8.5), 150 \mathrm{~mm} \mathrm{NaCl}, 5 \%$ glycerol, $5 \mathrm{~mm} 2-$ mercaptoethanol and $0.06 \% \quad N$-lauroyl sarcosine (NLS). The detergent was required because the solubility and the oligomeric state of pLG72 strongly depend upon the presence of NLS [17]. The area of each peak was estimated by nonlinear curve-fitting of the elution profile using PEAKFIT software (Systat Software, Erkrath, Germany). The amount of pLG72 and rDAAO present in the peak corresponding to the complex was estimated by means of the intensity of their bands after SDS/PAGE, as obtained using the software QUANTITY ONE (Bio-Rad, Hercules, CA, USA) and a known amount of purified rDAAO [17,21].

Limited proteolysis of rDAAO holo- and apoprotein forms $\left(0.4 \mathrm{mg} \cdot \mathrm{mL}^{-1}\right.$ protein) with $10 \%(\mathrm{w} / \mathrm{w})$ trypsin was 
carried out at $25^{\circ} \mathrm{C}$ in $20 \mathrm{~mm}$ Tris- $\mathrm{HCl}(\mathrm{pH} 8.5), 150 \mathrm{~mm}$ $\mathrm{NaCl}, 5 \%$ glycerol and $5 \mathrm{~mm}$ 2-mercaptoethanol as reported previously [23,37]. For the holoenzyme only, the experiment was also carried out in the presence of $1 \mathrm{~mm}$ benzoate or $0.1 \mathrm{~mm}$ FAD. The results are reported in Fig. S5 and Appendix S2.

\section{Partial purification of rDAAO from rat tissues}

Fresh rat kidneys were finely cut with a blade and homogenized in $10 \mathrm{~mm}$ Tris- $\mathrm{HCl}$ buffer $(\mathrm{pH} 7.4)$, containing $250 \mathrm{~mm}$ sucrose $\left(10 \mathrm{~mL} \cdot \mathrm{g}^{-1}\right.$ fresh kidney) with a homogenizer. The sample was centrifuged at $10000 \mathrm{~g}$ for $30 \mathrm{~min}$ at $4{ }^{\circ} \mathrm{C}$. As described previously [30], the supernatant was fractionated by adding $50 \%$ ammonium sulfate $(29.1 \mathrm{~g}$ per $100 \mathrm{~mL}$ of solution), followed by centrifugation at $39000 \mathrm{~g}$ for $1 \mathrm{~h}$ at $4{ }^{\circ} \mathrm{C}$. The precipitate was collected and dialyzed overnight against $20 \mathrm{~mm}$ sodium pyrophosphate buffer ( $\mathrm{pH}$ 8.3 ) and $10 \%$ glycerol.

\section{Detection of cellular D- and L-serine and rDAAO activity in U87-transfected cells}

The cDNA coding for rDAAO was inserted into the pEYFP-C3 vector (Clontech Laboratories, Palo Alto, CA, USA) using the HindIII and EcoRI restriction sites in-frame with the gene coding for EYFP. U87 Human glioblastoma cells (ATCC) were transfected by using the FuGENE HD Transfection reagent (Roche, Mannheim, Germany) in accordance with the manufacturer's instructions and as described previously [23]: $2 \mu \mathrm{g}$ of pEYFP-C3-rDAAO eukaryotic expression construct were used to transfect $2 \times 10^{5}$ cells per well. The protein expression level was monitored using a fluorescence microscope (Olympus IX51; Olympus America, Center Valley, PA, USA) equipped with a fluorescein isothiocyanate filter to detect the emission signal associated with the fluorescent tag and by western blot analysis using anti-actin-, anti-enhanced green fluorescent protein- or antiDAAO-specific sera. Stable clones were selected, adding $0.4 \mathrm{mg} \cdot \mathrm{mL}^{-1}$ of $\mathrm{G} 418$ to the growth medium [23].

The cellular D- and L-serine levels were determined by HPLC analysis as described previously [17]. Briefly, $\sim 2.5 \times 10^{5}$ U87-transfected cells were resuspended in $1 \mathrm{~mL}$ of ice-cold $5 \%$ trichloroacetic acid, sonicated and centrifuged for $30 \mathrm{~min}$ at $16000 \mathrm{~g}$. The soluble fraction was extracted with water-saturated dimethylether before precolumn derivatization with $o$-phthalaldehyde/ $N$-acetyl-L-cysteine in borate buffer. D- and L-serine were resolved by HPLC chromatography on a $5 \mu \mathrm{m}$ Waters $\mathrm{C} 8(4.6 \times 250 \mathrm{~mm})$ reverse phase column (Waters Corp., Milford, MA, USA) eluted under isocratic conditions using $0.1 \mathrm{M}$ sodium acetate buffer (pH 6.2) and $1 \%$ tetrahydrofuran at $1 \mathrm{~mL} \cdot \mathrm{min}^{-1}$ [17]. The compounds were identified on the basis of retention times and quantified from the peak areas. The analyses were replicated five times for each condition, and statistical anal- yses were performed using KALEIDAGRAPH software (Synergy Software, Reading, MA, USA).

DAAO activity on crude extracts of U87 control and EYFP-hDAAO or EYFP-rDAAO stably transfected cells was measured by using the Amplex Ultra-Red assay kit (Invitrogen, Karlsruhe, Germany) based on the detection of $\mathrm{H}_{2} \mathrm{O}_{2}$ by the peroxidase-mediated oxidation of the fluorogenic Amplex UltraRed dye [17,23]. Cells were suspended in ice-cold $50 \mathrm{~mm}$ sodium phosphate buffer $(\mathrm{pH} 7.4)$, containing $1 \mu \mathrm{M}$ pepstatin, $2 \mu \mathrm{M}$ leupeptin and $10 \mu \mathrm{M}$ FAD, sonicated for three cycles of $30 \mathrm{~s}$ each and centrifuged at $16000 \mathrm{~g}$ for $10 \mathrm{~min}$ at $4{ }^{\circ} \mathrm{C}$. An aliquot of these solutions $\left(0.75 \mathrm{~mL}\right.$ corresponding to $3 \times 10^{5}$ cells $)$ was diluted $1: 2$ in the activity assay solution containing $50 \mu \mathrm{M}$ Amplex UltraRed, 0.2 units $\cdot \mathrm{mL}^{-1}$ horseradish peroxidase, $10 \mathrm{mM} \mathrm{NaN}_{3}$, $10 \mu \mathrm{M}$ FAD and $50 \mathrm{~mm}$ D-alanine and incubated for $30 \mathrm{~min}$ at room temperature in the dark. The reactions were blocked by adding $20 \mu \mathrm{L}$ of Amplex UltraRed stop reagent, and the fluorescence emission was measured at $590 \mathrm{~nm}$. For each sample, controls without the substrate D-alanine and a sample to which $10 \mathrm{~mm}$ sodium benzoate (a well-known DAAO inhibitor) was added [1] were prepared. DAAO activity was expressed as the difference in fluorescence emission between the sample and control assay mixtures. A calibration curve was obtained by using known amounts of recombinant rDAAO (range 0-20 munits).

\section{Acknowledgements}

This work was supported by grants from MIUR prot. 2008N2FM4A_001 to L. Pollegioni, from Fondo di Ateneo per la Ricerca to L. Pollegioni, S. Sacchi and G. Molla, and from Fondazione CARIPLO to L. Pollegioni. We are grateful for the support of Consorzio Interuniversitario per le Biotecnologie and the Centro di Ricerca in Biotecnologie per la Salute Umana (Università degli studi dell'Insubria).

\section{References}

1 Pollegioni L, Piubelli L, Sacchi S, Pilone MS \& Molla G (2007) Physiological functions of D-amino acid oxidases: from yeast to humans. Cell Mol Life Sci 64, 1373-1394.

2 Pollegioni L \& Sacchi S (2010) Metabolism the neuromodulator D-serine. Cell Mol Life Sci 67, 2387-2404.

3 Millan MJ (2005) N-Methyl-D-aspartate receptors as a target for improved antipsychotic agents: novel insights and clinical perspectives. Psychopharmacology 179, $30-53$.

4 Coyle JT (2006) Glutamate and schizophrenia: beyond the dopamine hypothesis. Cell Mol Neurobiol 26, $365-384$. 
5 Hashimoto K, Fukushima T, Shimizu E, Komatsu N, Watanabe H, Shinoda N, Nakazato M, Kumakiri C, Okada S, Hasegawa H et al. (2003) Decreased serum levels of D-serine in patients with schizophrenia: evidence in support of the N-methyl-D-aspartate receptor hypofunction hypothesis of schizophrenia. Arch Gen Psychiatry 60, 572-576.

6 Madeira C, Freitas Me, Vargas-Lopez C, Wolosker H \& Panizzutti R (2008) Increased brain D-amino acid oxidase (DAAO) activity in schizophrenia. Schizophr Res 101, 76-83.

7 Burnet PW, Eastwood SL, Bristow GC, Godlewska BR, Sikka P, Walker M \& Harrison PJ (2008) D-amino acid oxidase activity and expression are increased in schizophrenia. Mol Psychiatry 13, 658-660.

8 Verrall L, Walker M, Rawlings N, Benzel I, Kew JN, Harrison PJ \& Burnet PW (2007) D-Amino acid oxidase and serine racemase in human brain: normal distribution and altered expression in schizophrenia. Eur J Neurosci 26, 1657-1669.

9 Tsai GE, Yang P, Chung LC, Tsai IC, Tsai CW \& Coyle JT (1999) D-Serine added to clozapine for the treatment of schizophrenia. Am J Psychiatry 156, 1822-1825.

10 Ferraris D, Duvall B, Ko YS, Thomas AG, Rojas C, Majer P, Hashimoto K \& Tsukamoto T (2008) Synthesis and biological evaluation of $\mathrm{D}$-amino acid oxidase inhibitors. J Med Chem 51, 3357-3359.

11 Adage T, Trillat AC, Quattropani A, Perrin D, Cavarec L, Shaw J, Guerassimenko O, Giachetti C, Gréco B, Chumakov I et al. (2008) In vitro and in vivo pharmacological profile of AS057278, a selective D-amino acid oxidase inhibitor with potential anti-psychotic properties. Eur Neuropsychopharmacol 18, 200-214.

12 Sparey T, Abeywickrema P, Almond S, Brandon N, Byrne N, Campbell A, Hutson PH, Jacobson M, Jones B, Munshi S et al. (2008) The discovery of fused pyrrole carboxylic acids as novel, potent Damino acid oxidase (DAO) inhibitors. Bioorg Med Chem Lett 18, 3386-3391.

13 Duplantier AJ, Becker SL, Bohanon MJ, Borzilleri KA, Chrunk BA, Downs J, Hu LY, El-Kattan A, James LC, Liu S et al. (2009) Discovery, SAR, and pharmacokinetics of a novel 3-hydroxyquinolin-2 $(1 \mathrm{H})$-one series of potent D-amino acid oxidase (DAAO) inhibitors. J Med Chem 52, 3576-3585.

14 Konno R (1998) Rat D-amino-acid oxidase cDNA: rat $\mathrm{D}$-amino-acid oxidase as an intermediate form between mouse and other mammalian D-amino-acid oxidases. Biochim Biophys Acta 1395, 165-170.

15 Romano D, Molla G, Pollegioni L \& Marinelli F (2009) Optimization of human D-amino acid oxidase in Escherichia coli. Protein Expr Purif 68, 72-78.

16 Molla G, Sacchi S, Bernasconi M, Pilone MS, Fukui K \& Pollegioni L (2006) Characterization of human D-amino acid oxidase. FEBS Lett 580, 23582364.

17 Sacchi S, Bernasconi M, Martineau M, Mothet JP, Ruzzene M, Pilone MS, Pollegioni L \& Molla G (2008) pLG72 modulates intracellular D-serine levels through its interaction with D-amino acid oxidase: effect on schizophrenia susceptibility. J Biol Chem 283, 22244-22256.

18 Gibson QH, Swoboda BEP \& Massey V (1964) Kinetics and mechanism of action of glucose oxidase. $J$ Biol Chem 259, 3927-3934.

19 Porter DJT, Voet JG \& Bright HJ (1977) Mechanistic features of the D-amino acid oxidase reaction studied by double stopped flow spectrophotometry. $J$ Biol Chem 252, 4464-4473.

20 Caldinelli L, Molla G, Sacchi S, Pilone MS \& Pollegioni L (2009) Relevance of weak flavin binding in human D-amino acid oxidase. Protein Sci 18, 801-810.

21 Casalin P, Pollegioni L, Curti B \& Pilone MS (1991) A study on apoenzyme from Rhodotorula gracilis D-amino acid oxidase. Eur J Biochem 197, 513-517.

22 Yagi K, Nagatsu T \& Ozawa T (1956) Free D-serine, $\mathrm{D}$-aspartate and D-alanine in central nervous system and serum in mutant mice lacking D-amino acid oxidase. Nature 177, 891-892.

23 Caldinelli L, Molla G, Bracci L, Lelli B, Pileri S, Cappelletti P, Sacchi S \& Pollegioni L (2010) Effect of ligand on human D-amino acid oxidase: implications for the development of new drugs for schizophrenia treatment. Protein Sci 19, 1500-1512.

24 Abbott A (2010) The drug deadlock. Nature 468, 158-159.

25 Smith SM, Uslaner JM \& Hutson PH (2010) The therapeutic potential of D-amino acid oxidase (DAAO) inhibitors. Open Med Chem J 27, 3-9.

26 Arnold G, Liscum L \& Holtzman E (1979) Ultrastructural localization of D-amino acid oxidase in microperoxisomes of the rat nervous system. $J$ Histochem Cytochem 27, 735-745.

27 Dememes D, Mothet JP \& Nicolas T (2006) Cellular distribution of $\mathrm{D}$-serine, serine racemase and $\mathrm{D}$-amino acid oxidase in the rat vestibular sensory epithelia. Neuroscience 137, 991-997.

28 Moreno S, Nardacci R, Cimini A \& Cerù MP (1999) Immunocytochemical localization of D-amino acid oxidase in rat brain. $J$ Neurocytol 28, 169-185.

29 Konno R, Okamura T, Kasai N, Summer KH \& Niwa A (2009) Mutant rat strain lacking D-amino acid oxidase. Amino Acids 37, 367-375.

30 Huynh MS, Horiike K, Tojo H, Katagiri M \& Yamano $\mathrm{T}$ (1985) Kinetic properties of rat kidney D-amino acid oxidase associated with peroxisomes. Comp Biochem Physiol B 80, 425-430.

31 Zhao W-J, Gao Z-Y, Wei H, Nie H-Z, Zhao Q, Zhou X-J \& Wang Y-X (2010) Spinal D-amino acid oxidase 
contributes to neuropathic pain in rats. $J$ Pharmacol Exp Ther 332, 248-254.

32 Pollegioni L, Falbo A \& Pilone MS (1992) Specificity and kinetics of Rhodotorula gracilis D-amino acid oxidase. Biochim Biophys Acta 1120, 11-16.

33 Pollegioni L, Langkau B, Tischer W, Ghisla S \& Pilone MS (1993) Kinetic mechanism of D-amino acid oxidases from Rhodotorula gracilis and Trigonopsis variabilis. J Biol Chem 268, 13850-13857.

34 Harris CM, Molla G, Pilone MS \& Pollegioni L (1999) Studies on the reaction mechanism of Rhodotorula gracilis D-amino-acid oxidase. J Biol Chem 274, 36233-36240.

35 Massey V (1991) A simple method for the determination of redox potentials. In Flavins and Flavoproteins (Curti B, Ronchi S \& Zanetti G eds), pp. 59-66. Walter de Gruyter \& Co, Berlin.

36 Pollegioni L, Porrini D, Molla G \& Pilone MS (2000) Redox potentials and their $\mathrm{pH}$ dependence of D-amino acid oxidase of Rhodotorula gracilis and Trigonopsis variabilis. Eur J Biochem 267, 6624-6632.

37 Caldinelli L, Iametti S, Barbiroli A, Bonomi F, Piubelli L, Ferranti P, Picariello G, Pilone MS \& Pollegioni L (2004) Unfolding intermediate in the peroxisomal flavoprotein D-amino acid oxidase. J Biol Chem 279, 28426-28434.

38 Frederiks WM, Van Noorden CJF, Marx F, Gallagher PT \& Swann BP (1993) In situ kinetic measurements of D-amino acid oxidase in rat liver with respect to its substrate specificity. Histochemical J 25, 578-582.

\section{Supporting information}

The following supplementary material is available:

Fig. S1. SDS/PAGE analysis of rDAAO purification from BL21(DE3)Star E. coli cells carrying the pET28rDAAO.

Fig. S2. Inhibition of recombinant rDAAO activity by benzoate.

Fig. S3. Inhibition of rDAAO partially purified from rat kidney by benzoate.

Fig. S4. Comparison of the model of rDAAO active site in complex with 3-hydroxyquinolin-2(1H)-one with the active site of hDAAO and of pkDAAO.

Fig. S5. Time course of trypsin digestion of different rDAAO forms.

Appendix S1. Redox properties of rDAAO.

Appendix S2. Limited proteolysis studies.

This supplementary material can be found in the online version of this article.

Please note: As a service to our authors and readers, this journal provides supporting information supplied by the authors. Such materials are peer-reviewed and may be re-organized for online delivery, but are not copy-edited or typeset. Technical support issues arising from supporting information (other than missing files) should be addressed to the authors. 